\title{
Comments on "Measuring memory for source: Some theoretical assumptions and technical limitations"
}

\author{
LINDA A. HENKEL \\ Princeton University, Princeton, New Jersey \\ and \\ NANCY FRANKLIN \\ State University of New York, Stony Brook New York
}

\begin{abstract}
Researchers must consider limitations and assumptions inherent in their measure of source monitoring when drawing conclusions, an important point raised by Murnane and Bayen (1998). However, the issues they raise do not invalidate the conclusions we draw from the findings reported in Henkel and Franklin (1998). Issues regarding conclusions about source monitoring performance, the relation between recognition and source accuracy, and the use of empirical and multinomial analyses are discussed.
\end{abstract}

Issues concerning proper measurement of source monitoring and the relation between source monitoring and recognition memory have been considered since Johnson's pioneering work (Johnson \& Raye, 1981), and recent discussions of these issues have helped to highlight the intricate relations among recognition, source monitoring, and various response biases (e.g., Batchelder \& Riefer, 1990; Johnson, Hashtroudi, \& Lindsay, 1993; Murnane \& Bayen, 1996). We agree wholeheartedly with Murnane and Bayen's (1998) principal point that researchers need to carefully consider limitations and assumptions inherent in their measure of source performance when drawing conclusions. We do, however, take issue with the implication that the data we reported do not sufficiently support the conclusions we draw from our experiments (Henkel \& Franklin, 1998).

Do we provide ample evidence, as we claim, that similarity of other experienced items can influence source judgments about a particular target memory? Our primary findings were higher source error rates for imagined items that physically resembled perceived items than for those with no physical similarity (Experiments $1-3$ ). We also found higher source error rates for imagined items that were conceptually related to perceived items than for those with no conceptual similarity (Experiment 2). The basic conclusion we draw from these findings is that judgments about a target memory's source can be influenced

We wish to thank Marcia Johnson, Xiangen Hu, Ron Kinchla, Scott Nolde, and Doreen De Leonardis for their helpful comments on this paper. Correspondence should be addressed to L. A. Henkel, Department of Psychology, University of North Florida, 4567 St. Johns Bluff Road South, Jacksonville, FL 32224 (e-mail: lhenkel@unf.edu)

—Accepted by previous editor, Geoffrey R. Loftus by information that is derived from other, specific nontarget events.

We agree with Murnane and Bayen's (1998) point that the source monitoring task we used (in which subjects decide whether each target item had been perceived, had been imagined, or was new) involves processes that may be used in both recognition and source discrimination and that therefore the primary analyses do not allow us to draw conclusions about the contribution that two theoretically distinct processes might make to source performance. Nevertheless, this observation does not challenge our basic conclusion regarding the impact of similarity on judgments of a target memory's source, nor does it reduce the importance of this finding. We can learn a great deal about how people determine the source of memories and how various factors influence such judgments through the measure we used to examine source monitoring performance. We can then use these findings to inform our models of memory and make and test predictions from those models. Issues that might be addressed include whether old/new recognition performance and source judgments are influenced similarly by various factors and what conditions lead to independent item recognition and source accuracy. We provided supplementary analyses to speculate on the role of item and source memory in source monitoring performance, and it is there that Murnane and Bayen's (1998) specific criticisms are primarily aimed.

How solid is the basis for our speculation? We noted in Henkel and Franklin (1998) that the reality monitoring errors we found cannot be based solely on differential recognition rates because source performance and recognition performance were affected differently by the manipulated variables. Murnane and Bayen (1998) correctly point out that our reported measure of recognition in- 
cluded performance on both old and new items, whereas our measure of source accuracy involved only old items. Importantly, however, when recognition rates for only old items are analyzed (i.e., the proportion of old items correctly recognized as old, namely perceived items called "perceived" or "imagined," and imagined items called "perceived" or "imagined"), we still find that recognition and source accuracy are differentially affected by source, pair type, and orienting task. For example, recognition for old items was higher for imagined than for perceived items, whereas source accuracy was lower for imagined than for perceived items. The patterns reported in notes 3,5 , and 7 of the Henkel and Franklin (1998) paper remain when new items are omitted from both source and recognition analyses. Thus, although Murnane and Bayen's (1998) point is valid as a general argument regarding data analysis, it does not provide a basis for concern about our results.

We reported multinomial analyses in appendices to the paper as an alternative way to assess recognition and source accuracy and to speculate on their role in source monitoring. Murnane and Bayen (1998) imply that these analyses were inappropriate because frequencies for new items were pooled across type of similarity. This pooling was done in Experiment 1 so that the key factors analyzed in the analysis of variance were analyzed in the multinomial models as well. Since similarity between old and new items was manipulated in Experiment 1 only, data were not pooled in this way for the other studies. We have conducted multinomial analyses with new items broken down by type of similarity for Experiment 1 , and they yielded similar patterns for the variables of interest.

Murnane and Bayen (1998) challenge the selection of Batchelder and Riefer's (1990) Model 6c for our supplemental data analyses. The primary reason we used this model was because it estimates parameters that should vary in ways that are meaningful and that are expected on the basis of the source monitoring framework (Johnson et al., 1993). Furthermore, the key comparisons involved contrasts between the same parameters across conditions rather than across parameters within the same condition. Although as Murnane and Bayen (1998) point out, Models $6 \mathrm{c}$ and $6 \mathrm{~b}$ provide equally good statistical fits for the data and thus do not allow us to ascertain whether the findings are due to differences in source discriminability per se or to differential guessing bias (see Batchelder \& Riefer, 1990), theoretical considerations (Johnson et al., 1993) and analysis of empirical data suggest that the results are more plausibly due to differences in source discriminability than to response bias. Empirical analyses showed that subjects were more likely to mistakenly claim that imagined items had been perceived than to claim that perceived items had been imagined, an asymmetry found in other studies as well (Johnson, Raye, Wang, \& Taylor, 1979; Johnson, Taylor, \&
Raye, 1977; Kahan \& Johnson, 1990). This does not appear to be based on an overall bias to respond "perceived," however, because new items were more likely to be falsely called "imagined" than "perceived." Although this too may reflect a general response bias (to call new items "imagined" when guessing as to their source), it cannot account for our primary finding for imagined items. In some situations the biases at play in guessing the source of new items may differ from those involved in guessing the source of old items (see Riefer, $\mathrm{Hu}, \&$ Batchelder, 1994), but there is no compelling reason to expect that to be the case here, especially given the relatively high similarity between new and old items. In addition, the rates of false alarms for new items were substantially lower than source error rates for old items, and when taken together, these findings suggest that the influence of other similar items on source judgments about a target memory is not due exclusively to recognition processes or guessing bias. Instead, information arising from the memories of other experienced items appears to affect source monitoring itself. Murnane and Bayen (1998) imply that our multinomial analyses may not have been justified, but if theoretically based assumptions and plausibility arguments cannot be invoked to justify a given multinomial model, then the multinomial approach would be quite limited in clarifying patterns in data.

Murnane and Bayen (1998) also raise the question whether our data satisfy the "recognition dominance assumption," which stipulates that conflicts between item memory decision processes and source memory decision processes must be resolved in favor of the item memory processes. That is, if a memory is judged to be new by item memory processes but is attributed to a particular source by source memory processes, the conflict must be resolved in favor of the item memory decision processes, rendering a response of "new" (see also Murnane \& Bayen, 1996). It is not clear what psychological state corresponds to such a conflict. Furthermore, the recognition dominance assumption is derived from a postulated model of memory in which recognition and source discriminability processes are assumed to be independent (Murnane \& Bayen, 1996). We have instead approached the issue with the view that although recognition and source identification may sometimes be dissociable, the various features associated with a memory upon which old/new and source judgments are based may have some overlap. This approach stems from the source monitoring framework and the large body of data put forth by Johnson and colleagues (1993). According to this model, source monitoring and recognition judgments may often draw on different aspects of memories and involve different cognitive processes, but these two judgments do not necessarily reflect two fundamentally different processes. It remains to be seen whether the most appropriate model of memory must include an assumption of 
recognition dominance, and thus it may not be the case that violations of the assumption are cause for concern about our data.

Furthermore, our data would still be informative even if the assumption were not met. Murnane and Bayen (1998) assert that under such conditions item and source memory are confounded in many measures of source monitoring (see also Murnane \& Bayen, 1996). We agree that this confounding may be problematic when conclusions specific to independent recognition detection and source discriminability processes are made. But it is not problematic when conclusions concern source monitoring as an attribution that may involve processes common to old/new recognition. While we of course agree with Murnane and Bayen's view that researchers must carefully consider measurement issues when drawing conclusions, it is only through further empirical research that we can determine how well various models of memory account for the influence of information derived from nontarget events on source judgments about a target memory.

\section{REFERENCES}

BATChELDER, W. H., \& RiefER, D. M. (1990). Multinomial models of source monitoring. Psychological Review, 97, 548-564.
Henkel, L. A., \& Franklin, N. (1998). Reality monitoring of physically similar and conceptually related objects. Memory \& Cognition, 26, 659-673.

Johnson, M. K., Hashtroudi, S., \& Lindsay, S. L. (1993). Source monitoring. Psychological Bulletin, 114, 3-28

Johnson, M. K., \& RaYE, C. L. (1981). Reality monitoring. Psychological Review, 88, 67-85.

Johnson, M. K., RaYe, C. L., WANG, A., \& TAYLoR, T. H. (1979). Fact and fantasy: The roles of accuracy and variability in confusing imaginations with perceptual experiences. Journal of Experimental Psychology: Human Learning \& Memory, 5, 229-240.

JoHnSON, M. K., TAYLOR, T. H., \& RAYE, C. L. (1977). Fact and fantasy The effects of internally generated events on the apparent frequency of externally generated events. Memory \& Cognition, 5, 116-122.

KaHAN, T. L., \& Johnson, M. K. (1990). Memory for seen and imagined rotations of alphanumeric characters. Journal of Mental Imagery, 14, 119-130.

Murnane, K., \& Bayen, U. J. (1996). An evaluation of empirical measures of source identification. Memory \& Cognition, 24, 417-428.

Murnane, K., \& Bayen, U. J. (1998). Measuring memory for source: Some theoretical assumptions and technical limitations. Memory \& Cognition, 26, 674-677.

Riefer, D. M., Hu, X., \& BATChelder, W. H. (1994). Response strategies in source monitoring. Journal of Experimental Psychology. Learning, Memory, \& Cognition, 20, 680-693.

(Manuscript received December 2, 1996; revision accepted January 7,1997 .) 